\title{
Adjustment of Preoperative Fasting Guidelines for Adult Patients Undergoing Elective Surgery
}

\author{
Eissa Bilehjani', Solmaz Fakhari1,2*, Samira Yavari ${ }^{3}$, Jafar Rahimi Panahi', \\ Mohammadreza Afhami1 , Bahman Nagipour ${ }^{1}$, Hojjat Pourfathi' ${ }^{1}$, Haleh Farzin ${ }^{1}$, \\ Ladan Javidi ${ }^{1}$ \\ ${ }^{1}$ Department of Anesthesiology, Tabriz University of Medical Sciences, Tabriz, Iran \\ ${ }^{2}$ Alzahra Hospital, Tabriz, Iran \\ ${ }^{3}$ Tabriz University of Medical Sciences, Tabriz, Iran \\ Email: "solmaz_fakhari@yahoo.com
}

Received 8 December 2015; accepted 27 December 2015; published 30 December 2015

Copyright (C) 2015 by authors and Scientific Research Publishing Inc.

This work is licensed under the Creative Commons Attribution International License (CC BY).

http://creativecommons.org/licenses/by/4.0/

(c) (7) Open Access

\section{Abstract}

Introduction: The typical order of nothing per oral (NPO) after midnight has been challenged in recent years, thus the American Society of Anesthesiology (ASA) revised in practice guidelines for preoperative fasting in healthy patients undergoing elective procedures, but many studies have showed that guidelines were not considered in clinical practice. The aim of this study was to evaluate the adjustment of preoperative NPO time with fasting guidelines in adult elective ophthalmic surgeries in a university educational ophthalmology hospital in Tabriz, Iran. Methods: In 3 months period, this descriptive study was conducted on 250 patients who scheduled for elective eye surgery. The investigator interviewed with patients before beginning of anesthesia and evaluated fasting duration for heavy meal, light meal and clear liquids and his or her satisfaction from NPO time and also investigated which staff recommended NPO time before anesthesia induction. Results: Fasting duration from heavy and light meal and clear liquids was 14.31 hours (8 - 23 hours), 12.46 hours (6 - 21 hours) and 11.54 hours ( 3 - 18 hours), respectively, that was not consistent with ASA guidelines. The discontent of the patients from prolonged NPO time was $60.8 \%$. The most complaint was thirsty $(42 \%)$. Ward nurses were the personnel who had the primary role in patients fasting time period $(47.6 \%)$. Conclusion: Preoperative fasting duration for heavy and light meal and clear liquids for elective ophthalmic surgery in this teaching hospital was very long and not consistent with ASA guidelines. Ward nurses had the major role in this malpractice. Thus it seems that personnel education may be very helpful.

${ }^{*}$ Corresponding author.

How to cite this paper: Bilehjani, E., Fakhari, S., Yavari, S., Panahi, J.R., Afhami, M., Nagipour, B., Pourfathi, H., Farzin, H. and Javidi, L. (2015) Adjustment of Preoperative Fasting Guidelines for Adult Patients Undergoing Elective Surgery. Open Journal of Internal Medicine, 5, 115-118. http://dx.doi.org/10.4236/ojim.2015.54016 


\section{Keywords}

\section{Elective Surgery, NPO, Preoperative Fasting}

\section{Introduction}

Fasting before general anesthesia is required. Decreasing of volume and acidity of gastric contents, therefore reducing risk of vomiting and pulmonary aspiration risk, known as Mendelson Syndrome, are the main proposal for fasting. It is routinely advised that patients must be fasted the night before surgery or at least 8 hours before operation, but numerous studies have showed that rate of vomiting and aspiration risk varies with different types of foods and liquids. However prolonged fasting can result in reducing of vomiting and aspiration risk, but also it may cause adverse side effects including dehydration, thirsty, hungry and resistance to insulin [1]. In other studies, rate of aspiration among normal persons and individual with systemic disease was reported 1 in 8000 [2]. No substantial bases exist for provided prolong fasting in preoperative period [3]. European nutrition and metabolism association recommended using of carbohydrate—rich liquids until 2 hours before surgery [4] [5]. In a study, average time of patients fasting in preoperational period was 12 - 24 hours and fasting times had clear differences with American Society of Anesthesiology's recommendations [6]. New guidelines advocated 2 hours fasting time for clear liquids, but 6 hours for milk and light meals before initiating of anesthesia [7]. Another study showed no differences among gastric acidity rate and contents volume of persons who were fasting all night and persons that drank water up to 3 hours before surgery [8]. Also aspiration pneumonia heavily causes morbidity and mortality; however it is a rare complication of general anesthesia [9]. Predisposing factors are morbid obesity, gastro esophageal reflux and esophagus motility disorders [10]. In placebo-controlled studies, preoperative use of histamine 2 receptors inhibitors was effective in reducing of gastric content volume and acidity [11] [12]. In our hospital, any appropriate guidelines do not exist for preoperative fasting time. Therefore, we decided to design this study thereby, to obtain essential information about fasting time in adults patients who candidate for eye surgery.

\section{Methods and Materials}

This descriptive study have been performed on 250 patients over 16 years old who undergone elective eye surgery from October 2014 through January 2014. These patients were visited the day before surgery by anesthesiologist who didn't intervene in data collecting process. Admitting to operating theater, they were enrolled consecutively to study and a prepared questionnaire was filled by investigators before surgery. Exclusion criteria from study included preoperative visit of patients by investigator, lack of patient's cooperation and satisfaction, memory and conscious disorders, patient unfavorable status for answer to questions, and finally emergency surgery. When entering to operating room, in few minutes, firstly the importance and common reason for preoperative fasting and probable complications due to stomach contents during anesthesia were discussed with the patients. The examples and consumption of different food groups (heavy meal, light meal and clear liquids) discussed. Then data due to fasting condition and any dissatisfaction questioned and collected. The effective persons in determination of the final fasting period were clarified. The collected data analyzed by Chi-squared relationship and Fisher's exact test or regression ratings.

\section{Results}

The mean age of patients was $60.32 \pm 17.50$ (max age $=94$ years, $\min =16$ years), $51.9 \%$ were male and $48.1 \%$ were female. The patients underwent a variety types of surgeries that most common of them with $76.4 \%$ was cataract surgery. Others types of surgeries included strabismus surgery, dacryocystoplasty, vitrectomy, silicon (prevalence $0.8 \%-8.8 \%$ ). In regression test, ranking odds ratio for cataract surgery patients compared with other surgical procedures were associated with more significant differences $(\mathrm{P}<0.0005)$. The prevalence of hypertension was higher by $34.4 \%$ prevalence than other underlying conditions. About $55.2 \%$ had a history of consuming one or more drugs in the week before surgery. In general, $60.8 \%$ of patients were dissatisfied with their fasting process. Chance of dissatisfaction to fasting process, significantly was high than satisfaction to it $(\mathrm{P}=$ 
0.001). The fasting time to heavy meal, light meal and clear liquids were $14.31 \pm 3.45$ hours (8 -23 hours), $12.46 \pm 2.66$ hours ( $6-21$ hours), $11.54 \pm 2.74$ hours ( $3-18$ hours), respectively. In general, $53.6 \%$ of patients previously did not be announced due to their operation time and $42 \%$ of patients believed that their operation has been done with delay. In majority of the patients (84.4\%), medical personals were responsible or involved in setting the time for fasting. However, in $10.8 \%$ of cases, in spite of medical team recommendation for suitable fasting time for different types of intakes, the patients denied any intake. In $4.8 \%$ of patients, there was not given any sufficient information to them, by personnel. In $47.6 \%$ ward nurses had the major role on the recommendation of long fasting period before surgery. On the recommendation for long fasting period, anesthesiologists and surgeons had a major role in only $3.6 \%$ and $6.8 \%$ of patients, respectively. The most common cause of patient dissatisfaction was the feeling of thirst (42\%) and hunger (37.6\%).

\section{Discussion}

Guideline of American Society of Anesthesiology recommended 2 - 4 hours fasting time for clear liquids, 6 hours semisolid foods and 8 hours for heavy meal. Also mentioned about drugs that patients can take their medication with up to 30 cc of water until two hours before surgery [7]. Cern Shaw et al. study in 2002 showed that patients candidate for elective surgery were fasting averaged 12 hours for liquid and 14 hours for solid food before surgery that significantly was longer than levels of fasting taught or advised. In $63 \%$ cases, patient education was performed by nurses and in $37 \%$ cases, by the doctor and nurse team [6]. Winslow et al. reported in the non-emergency surgical patients 12.5 hours perioperative fasting time for liquids and 14.2 hours for semi-solid and solid foods. Eighty five percent of them followed the non-food night guidelines, however, only $12 \%$ followed ASA guideline [13]. In the study of Kramer et al., direct face to face training or training videos associated with different results [14]. Miller and Wishart study, among patients who traditionally have followed the practice of fasting for 8 hours ago and patients who had a light breakfast 3 hours prior to surgery, in terms of volume, acidity of stomach contents and aspiration pneumonia did not show any difference [11]. Murphy et al were observed also same results in the aspiration between midnight and recommended unlimited consumption of liquids fasting until 3 hours before surgery [15]. Farya et al. found that reducing the amount of fasting before surgery lowers insulin resistance [16]. Oscar et al. reported 35\% of who had been fasting for more than 8 hours, complained of nausea and vomiting after surgery and those who received an hour before clear liquids, $18 \%$ complained of nausea and vomiting and feeling of thirst and hunger felt less [10]. In our study, period of fasting before surgery for a variety of food was more than the recommended time and it did not match with Anesthesiology Association America protocol [7]. However, as noted in our review of the fasting time, there was a lot of dissatisfaction. Similar other studies, in our study felt thirst was the most common complaint patients. Like them, the role of nurses in patient education for the vulnerable period of fasting before the procedure was dramatic, but in contrast, the role of nurse anesthesiology, surgeons and anesthesiologists was lower than that in these groups may have little relevance to practice and the need to increase the conversation. Since the duration of fasting before surgery in patients was more different with global standards, it necessitates more education to the medical team, particularly nurses. Kramer's study may as an example of direct instruction and even video as a viable alternative to brochures to show improvement in patient instructions. Our researches concerning the American society of Anesthesiology standards in the field fasting time before surgery were consistent previous study [14]. All other studies believe that long preoperative fasting time does not prevent not only from aspiration before surgery, but complications such as insulin resistance, nausea and vomiting after surgery is elevated [10]. Due to the long time of fasting, volume and acidity of stomach contents did not decrease in our study and the patients were dissatisfied because of long time of fasting before surgery, thus we can consider shorter preoperative fasting time [4]. In our study, the main causes of non-compliance with the appropriate preoperative fasting time included fasting after midnight, patients' traditionalism and lack of proper protocol in hospital. Since the time of our study, $15.6 \%$ of the patients were not aware of fasting time or consciously avoided of protocol, the need for the training is necessary. About the major problem in our hospitals, we do not know the exact time of fasting. In addition, most hospitals do not administer serum for patients at the time of fasting. In case of solve this problem, some of the most common complications of prolonged fasting, such as thirst will be resolved.

\section{Conclusion}

This study showed that preoperative fasting duration for heavy and light meal and clear liquids in elective oph- 
thalmic surgeries was very long and not consistent with ASA guidelines. Ward nurses had the primary role in this malpractice. Thus it seems that personnel education may be very helpful. It is recommended to perform studies in proposal to evaluate the effect of personnel education, especially ward nurses.

\section{References}

[1] Aguilar-Nascimento, J.E. and Dock-Nascimento, D.B. (2010) Reducing Preoperative Fasting Time: A Trend Based on Evidence. World Journal of Gastrointestinal Surgery, 2, 57-60.

[2] Warner, M.A., Warner, M.E. and Weber, J.G. (1993) Clinical Significance of Pulmonary Aspiration during the Perioperative Period. Anesthesiology, 78, 56-62.

[3] Ljungqvist, O. and Søreide, E. (2003) Preoperative Fasting. British Journal of Surgery, 90, 400-406.

[4] Weimann, A., Braga, M., Harsanyi, L., Laviano, A., Ljungqvist, O., Soeters, P., et al. (2006) ESPEN Guidelines on Enteral Nutrition: Surgery Including Organ Transplantation. Clinical Nutrition, 25, 224-244.

[5] Braga, M., Ljungqvist, O., Soeters, P., Fearon, K., Weimann, A. and Bozzetti, F. (2009) ESPEN Guidelines on Parenteral Nutrition: Surgery. Clinical Nutrition, 28, 378-386. http://dx.doi.org/10.1016/j.clnu.2009.04.002

[6] Crenshaw, J.T. and Winslow, E.H. (2002) Preoperative Fasting: Old Habits Die. AJN, 102, 36-44.

[7] American Society of Anesthesiologists Committee (2011) Practice Guidelines for Preoperative Fasting and the Use of Pharmacologic Agents to Reduce the Risk of Pulmonary Aspiration: Application to Healthy Patients Undergoing Elective Procedures: An Updated Report by the American Society of Anesthesiologists Committee on Standards and Practice Parameters. Anesthesiology, 114, 495-511.

[8] Maltby, J.R., Lewis, P., Martin, A. and Sutheriand, L.R. (1991) Gastric Fluid Volume and pH in Elective Patients Following Unrestricted Oral Fluid until Three Hours before Surgery. Canadian Journal of Anaesthesia, 38, 425-429.

[9] Maltby, J.R., Pytka, S., Watson, N.C., Cowan, R.A. and Fick, G.H. (2004) Drinking 300 mL of Clear Fluid Two Hours before Surgery Has No Effect on Gastric Fluid Volume and pH in Fasting and Non-Fasting Obese Patients. Canadian Journal of Anaesthesia, 51, 111-115.

[10] Miller, M., Wishart, H.Y. and Nimmo, W.S. (1997) Gastric Contents at Induction of Anesthesia. Is a 4-Hour Fast Necessary? British Journal of Anaesthesia, 55, 1185-1188. http://dx.doi.org/10.1093/bja/55.12.1185

[11] Exarhos, N.D., Logan, W.D., Abbott, O. and Hatcher, C.R. (1995) The Importance of pH and Volume in Tracheobronchial Aspiration. Chest; 47, 167-169.

[12] Kluger, M.T. and Short, T.G. (2003) Aspiration during Anesthesia: A Review of 133 Patients from Australian Anesthetic Incident Monitoring Study (AIMS). Anesthesia, 54, 19-26. http://dx.doi.org/10.1046/j.1365-2044.1999.00642.x

[13] Murphy, G.S., Ault, M.L., Wong, H.Y. and Szokol, J.W. (2000) The Effect of a New NPO Policy on Operating Room Utilization. Journal of Clinical Anesthesia, 12, 48-51.

[14] Kramer, F.M. (2000) Patient Perceptions of the Importance of Maintaining Preoperative NPO Status. AANA Journal, 68, 321-328.

[15] Crenshaw, J.T. and Winslow, E.H. (2008) Preoperative Fasting Duration and Medication Instruction: Are We Improving? AORN Journal, 88, 963-976.

[16] Faria, M.S., Aguilar-Nascimento, J.E., Pimenta, O.S., Alvarenga Jr., L.C., Dock-Nascimento, D.B. and Slhessarenko, N. (2009) Preoperative Fasting of 2 Hours Minimizes Insulin Resistance and Organic Response to Trauma after VideoCholecystectomy: A Randomized, Controlled, Clinical Trial. World Journal of Surgery, 33, 1158-1164. 